\title{
On a Characterization of Convergence in Banach Spaces with a Schauder Basis
}

\author{
Marat V. Markin (iD) and Olivia B. Soghomonian \\ Department of Mathematics, California State University, 5245 N. Backer Avenue, M/S PB 108, Fresno, CA 93740-8001, USA \\ Correspondence should be addressed to Marat V. Markin; mmarkin@csufresno.edu
}

Received 4 June 2021; Accepted 26 July 2021; Published 30 September 2021

Academic Editor: Atilla Gil Nyi

Copyright (c) 2021 Marat V. Markin and Olivia B. Soghomonian. This is an open access article distributed under the Creative Commons Attribution License, which permits unrestricted use, distribution, and reproduction in any medium, provided the original work is properly cited.

We extend the well-known characterizations of convergence in the spaces $l_{p}(1 \leq p<\infty)$ of $p$-summable sequences and $c_{0}$ of vanishing sequences to a general characterization of convergence in a Banach space with a Schauder basis and obtain as instant corollaries characterizations of convergence in an infinite-dimensional separable Hilbert space and the space $c$ of convergent sequences.

"The method in the present paper is abstract and is phrased in terms of Banach spaces, linear operators, and so on. This has the advantage of greater simplicity in proof and greater generality in applications."

Jacob T. Schwartz

\section{Introduction}

In normed vector spaces of sequences, termwise convergence, being a necessary condition for convergence of a sequence (of sequences), falls short of being characteristic (see, e.g., [1]). Thus, the natural question is as follows: what conditions are required to be, along with termwise convergence, necessary and sufficient for convergence of a sequence in such spaces?

It turns out that, in the Banach spaces $l_{p}(1 \leq p<\infty)$ of $p$-summable sequences with $p$-norm,

$$
x:=\left(x_{k}\right)_{k \in \mathbb{N}} \mapsto\|x\|_{p}:=\left[\sum_{k=1}^{\infty}\left|x_{k}\right|^{p}\right]^{1 / p},
$$

$\left(\mathbb{N}:=\{1,2, \ldots\}\right.$ is the set of natural numbers) and $c_{0}$ of vanishing sequences with $\infty$-norm,

$$
x:=\left(x_{k}\right)_{k \in \mathbb{N}} \mapsto\|x\|_{\infty}:=\sup _{k \in \mathbb{N}}\left|x_{k}\right|,
$$

only one additional condition is needed. The following characterizations of convergence in the foregoing spaces are well known.
Proposition 1 (characterization of convergence in $l_{p}$ $(1 \leq p<\infty))$. In the (real or complex) space $l_{p}(1 \leq p<\infty)$,

$$
\left(x_{k}^{(n)}\right)_{k \in \mathbb{N}}=: x^{(n)} \longrightarrow x:=\left(x_{k}\right)_{k \in \mathbb{N}}, \quad n \longrightarrow \infty,
$$

iff
(1) $\forall k \in \mathbb{N}: x_{k}^{(n)} \longrightarrow x_{k}, n \longrightarrow \infty$,
(2) $\forall \varepsilon>0 \exists K \in \mathbb{N} \forall n \in \mathbb{N}: \sum_{k=K+1}^{\infty}\left|x_{k}^{(n)}\right|^{p}<\varepsilon$. [1].

See, e.g., Proposition 2.16 in [2] and Proposition 2.17 in

Remarks 1

(i) Condition (1) is termwise convergence.

(ii) Condition (2) signifies the uniform convergence of the series,

$$
\sum_{k=1}^{\infty}\left|x_{k}^{(n)}\right|^{p}
$$

to their respective sums over $n \in \mathbb{N}$. 
Proposition 2 (characterization of convergence in $c_{0}$ ). In the (real or complex) space $c_{0}$,

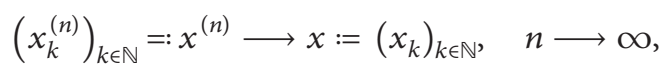

iff

$$
\begin{aligned}
& \text { (1) } \forall k \in \mathbb{N}: x_{k}^{(n)} \longrightarrow x_{k}, n \longrightarrow \infty, \\
& \text { (2) } \forall \varepsilon>0 \exists K \in \mathbb{N} \forall n \in \mathbb{N}: \sup _{k \geq K+1}\left|x_{k}^{(n)}\right|<\varepsilon .
\end{aligned}
$$
[1].

See, e.g., Proposition 2.15 in [2] and Proposition 2.16 in

\section{Remarks 2}

(i) Condition (1) is termwise convergence.

(ii) Condition (2) signifies the uniform convergence of the sequences $\left(x_{k}^{(n)}\right)_{k \in \mathbb{N}}$ to 0 over $n \in \mathbb{N}$.

One cannot but notice that both characterizations share the same condition (1) and that condition (2) in each can be reformulated in the following equivalent form:

(2C) $\forall \varepsilon>0 \exists K_{0} \in \mathbb{N} \forall K \geq K_{0} \forall n \in \mathbb{N}:\left\|R_{K} x^{(n)}\right\|<\varepsilon$,

where $\|\cdot\|$ stands for $p$-norm $\|\cdot\|_{p}(1 \leq p<\infty)$ or $\infty$-norm, respectively, and the mapping $R_{K}: X \longrightarrow X$, $K \in \mathbb{N},\left(X:=l_{p}(1 \leq p<\infty)\right.$ or $\left.X:=c_{0}\right)$ is defined as follows: $x:=\left(x_{k}\right)_{k \in \mathbb{N}} \mapsto R_{K} x:=(\underbrace{0, \ldots, 0}_{K \text { terms }}, x_{K+1}, x_{K+2}, \ldots), \quad K \in \mathbb{N}$.

Thus, we have the following combined characterization encompassing both $l_{p}(1 \leq p<\infty)$ and $c_{0}$.

Proposition 3 (combined characterization of convergence). In the (real or complex) space $X:=l_{p}(1 \leq p<\infty)$ or $X:=c_{0}$,

$$
\left(x_{k}^{(n)}\right)_{k \in \mathbb{N}}=: x^{(n)} \longrightarrow x:=\left(x_{k}\right)_{k \in \mathbb{N}}, \quad n \longrightarrow \infty,
$$

iff

$$
\begin{aligned}
& \text { (1) } \forall k \in \mathbb{N}: x_{k}^{(n)} \longrightarrow x_{k}, n \longrightarrow \infty, \\
& \text { (2C) } \forall \varepsilon>0 \exists K_{0} \in \mathbb{N} \forall K \geq K_{0} \forall n \in \mathbb{N}:\left\|R_{K} x^{(n)}\right\|<\varepsilon,
\end{aligned}
$$

where $\|\cdot\|$ stands for $p$-norm $\|\cdot\|_{p}(1 \leq p<\infty)$ or $\infty$-norm, respectively, and the mapping $R_{K}: X \longrightarrow X, K \in \mathbb{N}$, is defined by (6).

In view of the fact that both $l_{p}(1 \leq p<\infty)$ and $c_{0}$ are Banach spaces with a Schauder basis, our goal to show that a two-condition characterization of convergence, similar to the foregoing combined characterization, holds for all such spaces appears to be amply motivated. We establish a general characterization of convergence in a Banach space with a Schauder basis and obtain as instant corollaries characterizations of convergence in an infinite-dimensional separable Hilbert space and the Banach space $c$ of convergent sequences.

\section{Preliminaries}

Here, we briefly outline certain preliminaries essential for our discourse.
Definition 1 (Schauder basis). A Schauder basis (also a countable basis) of a (real or complex) Banach space $(X,\|\cdot\|)$ is a countably infinite set $\left\{e_{n}\right\}_{n \in \mathbb{N}}$ in $X$ such that

$$
\forall x \in X \exists !\left(c_{k}(x)\right)_{k \in \mathbb{N}} \in \mathbb{F}^{\mathbb{N}}: x=\sum_{k=1}^{\infty} c_{k}(x) e_{k},
$$

$(\mathbb{F}:=\mathbb{R}$ or $\mathbb{F}:=\mathbb{C})$ the series called the Schauder expansion of $x$ and the numbers $c_{k}(x) \in \mathbb{F}, k \in \mathbb{N}$, the coordinates of $x$ relative to $\left\{e_{n}\right\}_{n \in \mathbb{N}}$.

See, e.g., [1-4].

For an infinite-dimensional separable Hilbert space $(X,(\cdot, \cdot),\|\cdot\|)((\cdot, \cdot)$ stands for inner product and $\|\cdot\|$ for inner product norm), an orthonormal basis $\left\{e_{n}\right\}_{n \in \mathbb{N}}$ is a Schauder basis, and for an arbitrary $x \in X$,

$$
x=\sum_{k=1}^{\infty} c_{k}(x) e_{k}, \quad \text { with } c_{k}(x)=\left(x, e_{k}\right), k \in \mathbb{N},
$$

(see, e.g., $[1,2]$ ).

As we mention above, the sequence spaces $l_{p}$ $(1 \leq p<\infty), c_{0}$, and $c$ are examples of Banach spaces with a Schauder basis. For $l_{p}(1 \leq p<\infty)$ and $c_{0}$, the standard Schauder basis is the set

$$
\left\{e_{n}:=\left(\delta_{n k}\right)_{k \in \mathbb{N}}\right\}_{n \in \mathbb{N}}
$$

$\left(\delta_{n k}\right.$ is the Kronecker delta) and for an arbitrary $x:=\left(x_{k}\right)_{k \in \mathbb{N}}$ in the foregoing spaces,

$$
x=\sum_{k=1}^{\infty} c_{k}(x) e_{k}, \quad \text { with } c_{k}(x)=x_{k}, k \in \mathbb{N}
$$

(see, e.g., [1-4]).

For the Banach space $c$ of convergent sequences equipped with $\infty$-norm (see (2)), the standard Schauder basis is $\left\{e_{n}\right\}_{n \in \mathbb{Z}_{+}}\left(\mathbb{Z}_{+}:=\{0,1,2, \ldots\}\right.$ is the set of nonnegative integers) with

$$
e_{0}:=(1,1,1, \ldots)
$$

and for an arbitrary $x:=\left(x_{k}\right)_{k \in \mathbb{N}} \in c$,

$$
\begin{aligned}
x & =\sum_{k=0}^{\infty} c_{k}(x) e_{k}, \quad \text { with } c_{0}(x)=\lim _{m \longrightarrow \infty} x_{m}, c_{k}(x) \\
& =x_{k}-c_{0}(x), k \in \mathbb{N},
\end{aligned}
$$

see, e.g., [1-4].

Banach spaces with more sophisticated Schauder bases encompass $L_{p}(a, b) \quad(1 \leq p<\infty)$ and $C[a, b] \quad(-\infty<a<$ $b<\infty$ ) with $\infty$-norm,

$$
C[a, b] \ni x \mapsto\|x\|_{\infty}:=\max _{a \leq t \leq b}|x(t)|
$$

(see, e.g., $[3,4])$.

A Banach space with a Schauder basis is infinite-dimensional and separable (see, e.g., [1-3]). However, an infinite-dimensional separable Banach space need not have a Schauder basis (see [5]).

The set of $\mathbb{F}$-termed sequences, 


$$
Y:=\left\{y:=\left(c_{k}\right)_{k \in \mathbb{N}} \in \mathbb{F}^{\mathbb{N}} \mid \sum_{k=1}^{\infty} c_{k} e_{k} \text { converges in } X\right\},
$$

with termwise linear operations and the norm,

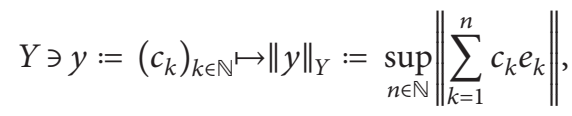

is a Banach space and the linear operator,

$$
Y \ni y:=\left(c_{k}\right)_{k \in \mathbb{N}} \mapsto A y:=\sum_{k=1}^{\infty} c_{k} e_{k} \in X,
$$

is subject to the inverse mapping theorem (see, e.g., $[1-3,6]$ ). The boundedness of the inverse operator $A^{-1}: X \longrightarrow Y$ implies boundedness, and hence, continuity, for the linear Schauder coordinate functionals,

$$
X \ni x=\sum_{k=1}^{\infty} c_{k}(x) e_{k} \mapsto c_{n}(x) \in \mathbb{F}, \quad n \in \mathbb{N},
$$

with

$$
\left\|c_{n}\right\| \leq \frac{2\left\|A^{-1}\right\|}{\left\|e_{n}\right\|}, \quad n \in \mathbb{N},
$$

(see, e.g., $[1-3]$ ) as well as for the linear operators:

$$
\begin{aligned}
X \ni x & =\sum_{k=1}^{\infty} c_{k}(x) e_{k} \mapsto S_{n} x:=\sum_{k=1}^{n} c_{k}(x) e_{k}, \\
R_{n} x & :=\sum_{k=n+1}^{\infty} c_{k}(x) e_{k}, \quad n \in \mathbb{N},
\end{aligned}
$$

with

$$
I=S_{n}+R_{n}, \quad n \in \mathbb{N},
$$

( $I$ is the identity operator on $X$ ) and

$$
\left\|S_{n}\right\| \leq\left\|A^{-1}\right\|,\left\|R_{n}\right\| \leq 2\left\|A^{-1}\right\|, \quad n \in \mathbb{N},
$$

(see, e.g., [3]).

Remark 3. Here and henceforth, we use the notation $\|\cdot\|$ for the operator norm.

\section{General Characterization}

The following statement appears to be a perfect illustration of the profound observation by Schwartz found in [7] and chosen as the epigraph.

Theorem 1 (general characterization of convergence). Let $(X,\|\cdot\|)$ be a (real or complex) Banach space with a Schauder basis $\left\{e_{n}\right\}_{n \in \mathbb{N}}$ and corresponding coordinate functionals $c_{n}(\cdot)$, $n \in \mathbb{N}$.

For a sequence $\left(x_{n}\right)_{n \in \mathbb{N}}$ and a vector $x$ in $X$,

$$
x_{n} \longrightarrow x, \quad n \longrightarrow \infty
$$

iff

(1) $\forall k \in \mathbb{N}: c_{k}\left(x_{n}\right) \longrightarrow c_{k}(x), n \longrightarrow \infty$,

(2) $\forall \varepsilon>0 \exists K_{0} \in \mathbb{N} \forall K \geq K_{0} \forall n \in \mathbb{N}:\left\|R_{K} x_{n}\right\|<\varepsilon$.

Proof. "Only if" part.

Suppose that, for a sequence $\left(x_{n}\right)_{n \in \mathbb{N}}$ and a vector $x$ in $X$,

$$
x_{n} \longrightarrow x, \quad n \longrightarrow \infty \text {. }
$$

Then, by the continuity of the Schauder coordinate functionals $c_{n}(\cdot), n \in \mathbb{N}$, we infer that condition (1) holds.

Let $\varepsilon>0$ be arbitrary. Then,

$$
\exists N \in \mathbb{N} \forall n \geq N:\left\|x_{n}-x\right\|<\frac{\varepsilon}{4\left\|A^{-1}\right\|} .
$$

Since $x \in X$,

$$
R_{K} x:=\sum_{k=K+1}^{\infty} c_{k}(x) e_{k} \longrightarrow 0, \quad K \longrightarrow \infty,
$$

and hence,

$$
\exists K_{0} \in \mathbb{N} \forall K \geq K_{0}:\left\|R_{K} x\right\|<\frac{\varepsilon}{2} .
$$

In view of (22), (25), and (27), we have

$$
\begin{aligned}
\forall K & \geq K_{0}, \forall n \geq N:\left\|R_{K} x_{n}\right\|=\left\|R_{K} x_{n}-R_{K} x+R_{K} x\right\| \\
& \leq\left\|R_{K}\left(x_{n}-x\right)\right\|+\left\|R_{K} x\right\| \\
& \leq\left\|R_{K}\right\|\left\|x_{n}-x\right\|+\left\|R_{K} x\right\| \\
& <2\left\|A^{-1}\right\| \frac{\varepsilon}{4\left\|A^{-1}\right\|}+\frac{\varepsilon}{2}=\frac{\varepsilon}{2}+\frac{\varepsilon}{2}=\varepsilon .
\end{aligned}
$$

Furthermore, since $x_{n} \in X, n=1, \ldots, N-1$, we can regard $K_{0} \in \mathbb{N}$ in (27) to be large enough so that

$$
\forall K \geq K_{0}, \forall n=1, \ldots, N-1:\left\|R_{K} x_{n}\right\|=\left\|\sum_{k=K+1}^{\infty} c_{k}\left(x_{n}\right) e_{k}\right\|<\varepsilon .
$$

Thus, condition (2) holds as well.

This completes the proof of the "only if" part.

"If" part. Suppose that, for a sequence $\left(x_{n}\right)_{n \in \mathbb{N}}$ and a vector $x$ in $X$, conditions (1) and (2) are met.

For an arbitrary $\varepsilon>0$ and $K_{0} \in \mathbb{N}$, from condition (2), by condition (1),

$$
\exists N \in \mathbb{N} \forall n \geq N:\left\|S_{K_{0}}\left(x_{n}-x\right)\right\| \leq \sum_{k=1}^{K_{0}}\left|c_{k}\left(x_{n}\right)-c_{k}(x)\right|\left\|e_{k}\right\|<\frac{\epsilon}{3} .
$$

Since $x \in X$, we can also regard that $K_{0} \in \mathbb{N}$ in condition (2) to be large enough so that

$$
\left\|R_{K_{0}} x\right\|<\frac{\varepsilon}{3} .
$$


Then, in view of (21), (30), and (31) and by condition (2),

$$
\begin{aligned}
\forall n & \geq N:\left\|x_{n}-x\right\|=\left\|S_{K_{0}}\left(x_{n}-x\right)+R_{K_{0}}\left(x_{n}-x\right)\right\| \\
& \leq\left\|S_{K_{0}}\left(x_{n}-x\right)\right\|+\left\|R_{K_{0}} x_{n}\right\|+\left\|R_{K_{0}} x\right\| \\
& <\frac{\varepsilon}{3}+\frac{\varepsilon}{3}+\frac{\varepsilon}{3}=\varepsilon .
\end{aligned}
$$

This concludes the proof of the "if" part and the entire statement.

Remarks 4

(i) Condition (1) is the convergence of the coordinates of $x_{n}$ to the corresponding coordinates of $x$ relative to $\left\{e_{k}\right\}_{k \in \mathbb{N}}$.

(ii) Condition (2) signifies the uniform convergence of the Schauder expansions,

$$
\sum_{k=1}^{\infty} c_{k}\left(x_{n}\right) e_{k}
$$

of $x_{n}$ relative to $\left\{e_{k}\right\}_{k \in \mathbb{N}}$ over $n \in \mathbb{N}$.

Now, the combined characterization of convergence (Proposition 3) is an instant corollary of the foregoing general characterization.

\section{Characterization of Convergence in an Infinite-Dimensional Separable Hilbert Space}

For an infinite-dimensional separable Hilbert space $(X,(\cdot, \cdot),\|\cdot\|)$ relative to an orthonormal basis $\left\{e_{n}\right\}_{n \in \mathbb{N}}$, in view of (9), the general characterization of convergence (Theorem 1) acquires the following form.

Corollary 1 (characterization of convergence in a separable Hilbert space). Let $(X,(\cdot, \cdot),\|\cdot\|)$ be a (real or complex) infinite-dimensional separable Hilbert space with an orthonormal basis $\left\{e_{n}\right\}_{n \in \mathbb{N}}$.
For a sequence $\left(x_{n}\right)_{n \in \mathbb{N}}$ and a vector $x$ in $X$,

$$
x_{n} \longrightarrow x, \quad n \longrightarrow \infty \text {, }
$$

iff

(1) $\forall k \in \mathbb{N}:\left(x_{n}, e_{k}\right) \longrightarrow\left(x, e_{k}\right), n \longrightarrow \infty$,

(2) $\forall \varepsilon>0 \exists K \in \mathbb{N} \forall n \in \mathbb{N}: \sum_{k=K+1}^{\infty}\left|\left(x_{n}, e_{k}\right)\right|^{2}<\varepsilon$.

Remarks 5

(i) Condition (1) is the convergence of the Fourier coefficients of $x_{n}$ to the corresponding Fourier coefficients of $x$ relative to $\left\{e_{k}\right\}_{k \in \mathbb{N}}$.

(ii) Condition (2) signifies the uniform convergence of the Fourier series expansions,

$$
\sum_{k=1}^{\infty}\left(x_{n}, e_{k}\right) e_{k},
$$

of $x_{n}$ relative to $\left\{e_{k}\right\}_{k \in \mathbb{N}}$ over $n \in \mathbb{N}$.

(iii) The characterization of convergence in $l_{p}$ (Proposition 1) for $p=2$ is now a particular case of the prior characterization.

\section{Characterization of Convergence in $c$}

Another immediate corollary of the general characterization of convergence (Theorem 1) is the realization of the latter in the space $c$ of convergent sequences equipped with $\infty$-norm (see (2)) relative to the standard Schauder basis $\left\{e_{n}\right\}_{n \in \mathbb{Z}_{+}}$(see Section 2).

Indeed, in $c$ relative to $\left\{e_{n}\right\}_{n \in \mathbb{Z}_{+}}$, for an arbitrary $x:=\left(x_{k}\right)_{k \in \mathbb{N}}$,

$$
\begin{aligned}
x & =\sum_{k=0}^{\infty} c_{k}(x) e_{k}, \quad \text { with } c_{0}(x)=\lim _{m \longrightarrow \infty} x_{m}, c_{k}(x) \\
& =x_{k}-c_{0}(x), k \in \mathbb{N},
\end{aligned}
$$

(see (13)) and

$$
\begin{aligned}
& S_{K} x:=\sum_{k=0}^{K} c_{k}(x) e_{k}=\left(\lim _{m \longrightarrow \infty} x_{m}, x_{1}-\lim _{m \longrightarrow \infty} x_{m}, \ldots, x_{K}-\lim _{m \longrightarrow \infty} x_{m}, 0, \ldots\right), \\
& R_{K} x:=\sum_{k=K+1}^{\infty} c_{k}(x) e_{k}=(\underbrace{0, \ldots, 0}_{K+1 \text { terms }}, x_{K+1}-\lim _{m \longrightarrow \infty} x_{m}, \ldots), \quad K \in \mathbb{Z}_{+},
\end{aligned}
$$

(cf. (20)).

Thus, the general characterization of convergence (Theorem 1), in view of the obvious circumstance that, for any $x:=\left(x_{k}\right)_{k \in \mathbb{N}} \in c$, the sequence,

$$
\left\|R_{K} x\right\|=\sup _{k \geq K+1}\left|x_{k}^{(n)}-\lim _{m \longrightarrow \infty} x_{m}^{(n)}\right|, \quad K \in \mathbb{Z}_{+},
$$

is decreasing, acquires the following form.

Corollary 2 (characterization of convergence in $c$ ). In the (real or complex) space $c$,

$$
\left(x_{k}^{(n)}\right)_{k \in \mathbb{N}}=: x^{(n)} \longrightarrow x:=\left(x_{k}\right)_{k \in \mathbb{N}}, \quad n \longrightarrow \infty,
$$


iff

(1) $\lim _{m \longrightarrow \infty} x_{m}^{(n)} \longrightarrow \lim _{m \longrightarrow \infty} x_{m}, \quad n \longrightarrow \infty, \quad$ and
$\forall k \in \mathbb{N}: x_{k}^{(n)} \longrightarrow x_{k}, n \longrightarrow \infty$,
(2) $\forall \varepsilon>0 \exists K \in \mathbb{Z}_{+} \forall n \in \mathbb{N}: \sup _{k \geq K+1}\left|x_{k}^{(n)}-\lim _{m \longrightarrow \infty} x_{m}^{(n)}\right|<\varepsilon$.

Remarks 6

(i) Condition (1), beyond termwise convergence, includes convergence of the limits.

(ii) Condition (2) signifies the uniform convergence of the sequences $\left(x_{k}^{(n)}\right)_{k \in \mathbb{N}}$ to their respective limits over $n \in \mathbb{N}$.

(iii) The characterization of convergence in $c_{0}$ (Proposition 2) is a mere restriction of the prior characterization to the subspace $c_{0}$ of $c$.

\section{Concluding Remark}

As is easily seen, the general characterization of convergence (Theorem 1) is consistent with the following characterization of compactness, which underlies the results of [8].

Theorem 2 (characterization of compactness, Theorem III.7.4 in [3]). In a (real or complex) Banach space $(X,\|\cdot\|)$ with a Schauder basis, a set $C$ is precompact (a closed set $C$ is compact) iff

(1) $C$ is bounded,

(2) $\forall \varepsilon>0 \exists K_{0} \in \mathbb{N} \forall K \geq K_{0} \forall x \in C:\left\|R_{K} x\right\|<\varepsilon$.

\section{Data Availability}

No data were used to support this study.

\section{Conflicts of Interest}

The authors declare that they have no conflicts of interest.

\section{References}

[1] M. V. Markin, Elementary Operator Theory, De Gruyter Graduate, Walter de Gruyter GmbH, Berlin, Germany, 2020.

[2] M. V. Markin, Elementary Functional Analysis, De Gruyter Graduate, Walter de Gruyter GmbH, Berlin, Germany, 2018.

[3] L. A. Lyusternik and V. I. Sobolev, A Short Course in Functional Analysis, Vysshaya Shkola, Moscow, Russia, 1982.

[4] I. Singer, Bases in Banach Spaces. I, Die Grundlehren der Mathematischen Wissenschaften, Band 154, Springer-Verlag, Berlin, Germany, 1970.

[5] P. Enflo, "A counterexample to the approximation problem in Banach spaces," Acta Mathematica, vol. 130, pp. 309-317, 1973.

[6] N. Dunford and J. T. Schwartz with the assistance of W.G. Bade and R.G. Bartle, Linear Operators. Part I: General Theory, Interscience Publishers, New York, NY, USA, 1958.

[7] J. Schwartz, "Perturbations of spectral operators, and applications. I. Bounded perturbations," Pacific Journal of Mathematics, vol. 4, no. 3, pp. 415-458, 1954.
[8] M. V. Markin, "On the strong continuity of functions defined on a compact set with values in a Banach space with a basis," Mathematics Today '90, no. 6, pp. 110-118, Vishcha Shkola, Kiev, Ukraine, 1990. 